\title{
A Through Wall Doppler Radar System: Active Textile Antenna Design, Prototyping and Experiment
}

\author{
Sam Agneessens, Patrick Van Torre, Frederick Declercq, Bart Spinnewyn, Gert-Jan Stockman, \\ Hendrik Rogier, Dries Vande Ginste \\ Department of Information Technology, Ghent University, Sint-Pietersnieuwstraat 41, 9000 Ghent, \\ Belgium
}

\begin{abstract}
Using garments as a platform for electronic sensing and communication systems opens up a wide range of novel and exciting applications. By carefully tailoring the antenna properties and by adopting a dedicated design strategy, a robust wearable antenna system can be obtained onto which all necessary electronics are integrated. In this contribution, the dedicated design for approach of a lowweight, wearable Doppler radar system fabricated on textile materials is presented. The system, fully integrated into a rescue worker's garment, is capable of detecting moving objects behind a barrier. It relies on an array of four textile transmit antennas to scan the surroundings. At the receiving end, an active wearable receive antenna is deployed to capture the reflected signals. It is demonstrated that the on-body system is capable of detecting moving subjects in indoor environments, including through-wall scenarios.
\end{abstract}

Index Terms - Wearable wireless sensors, active textile antennas, Doppler radar

\section{INTRODUCTION}

Rescue personnel, firefighters or law enforcement officials are often required to access difficult or hostile terrain and during the completion their assigned task, their own safety is primordial. The use of wireless sensors and systems (communications, vital sign monitoring, etc.) can tip the balance between success and failure.

The gear typically used in rescue operations consists of protective garments (to shield the wearer from water, intense heat, etc.), helmet, breathing equipment, tools, etc. and, hence, is very heavy. This makes extension of the functionality with additional standalone equipment unpractical to carry along, as it weighs down and limits the user's movements, resulting in more hinder than help.

A functionality that would be useful in security and rescue applications is the ability to detect and track moving objects through closed doors and walls [1]. To be deployable in realistic situations, it is vital that such a radar system is compact, low-weight and comfortable to wear [2].

Over the last years, developments in the field of textile antennas have seen that designs have evolved from a standalone component to a complete system. Integration of active electronics circuitry and construction of complete wearable arrays, have paved the way for novel body-centric applications, where the antenna module is not just a simple communication device anymore.

In this contribution, it is demonstrated how a dedicated design formalism combined with a suitable manufacturing process allows an extension of the functionality of the rescue personnel's gear by using the materials available in the protective garments, rather than adding an extra piece of equipment. Smart textiles are used for the integration of a radar system into protective garments, which allows the wearer to detect moving targets behind a barrier and adds little extra weight.

The paper is organized as follows: in section II the used textile materials are described, section III discusses the radar's different subsystems. Measurements and results are presented in section IV and conclusions in Section V.

\section{PROTECTIVE GARMENTS AS EM SUBSTRATES}

Off-the-shelf textile materials, found in protective garments, are used as textile substrate for the active circuitry and antenna design. This allows for easy integration of the completed system into the garments. Efficient and correct design requires accurate knowledge of the electromagnetic properties of the substrates, but unlike classical highfrequency laminates, material characteristics such as permittivity and losses are not readily available and a dedicated characterization technique for textile antennas is used to extract these parameters [3].

\section{A. Aramid}

A textile material typically used as the outer layer in firefighter jackets, it is flame resistant, fire retardant, non conductive. The fabric is twill woven from identical yarns, resulting in an even surface. EM characterization yields a permittivity er $=1.69$ and low losses: $\tan \delta=0.015$. These properties, along with a small thickness of $200 \mu \mathrm{m}$, make the aramid textile well suited for use as a carrier for electronic circuits.

\section{B. Polyurethane foam}

Polyurethane foam manufactured by Brunet Lion called Azzurri is used in the production of firefighter jackets. It is fire retardant and has electromagnetic properties which are relatively insensitive to changes in relative humidity [4]. 
Measured $\mathrm{e}_{\mathrm{r}}=1.19, \tan \delta=0.003$ and thickness of $3.55 \mathrm{~mm}$ make the foam well suited to be used as an antenna substrate.

\section{RADAR SYSTEM DESIGN}

The key components of the wearable radar system, being the active and passive textile antennas, are fabricated from flexible, low-weight materials commonly found in protective garments. These elements are deployed in an on-body radar system with a basic Doppler architecture. The system's operating frequency is $2.35 \mathrm{GHz}$, where wall penetration is possible and interference from the $2.45 \mathrm{GHz}$ Industrial, Medical and Scientific (ISM) band is avoided.

\section{A. Transmit Side}

At the transmit side the large on-body area is exploited to integrate a 4-element phased array, capable of scanning the surroundings. Each individual antenna has a rectangular ring topology with probe feed and is designed to be right handed circularly polarized (RHCP). The conductive antenna parts (i.e. ground plane and patch radiator) are fabricated with the e-textile Flectron ${ }^{\circledR}$ and are attached to the polyurethane foam antenna substrate by means of an adhesive sheet. Array topology and element dimensions are shown in Fig. 1.

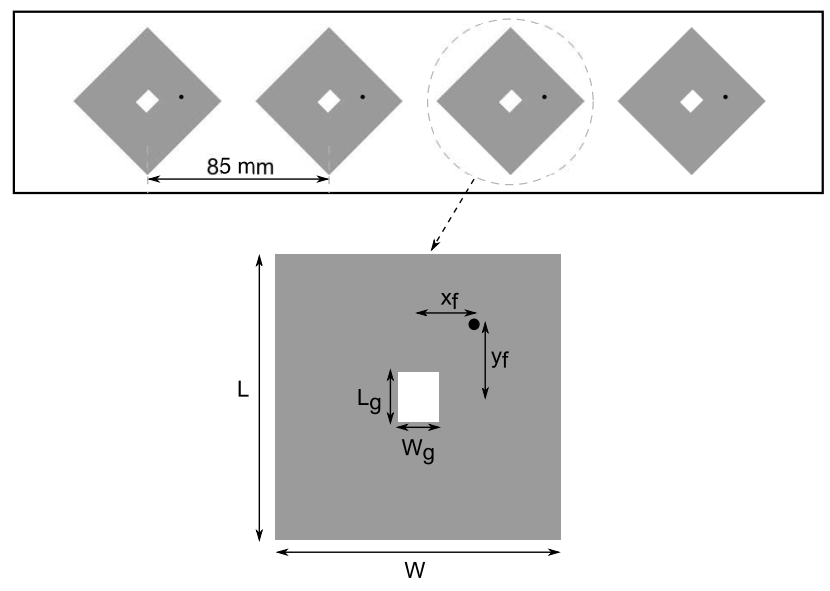

Fig. 1. Passive transmit array topology $\left(\mathrm{W}=51.8 \mathrm{~mm}, \quad \mathrm{~L}=55.4 \mathrm{~mm}, \quad \mathrm{~W}_{\mathrm{g}}=8.8 \mathrm{~mm}\right.$, $\left.\mathrm{L}_{\mathrm{g}}=7.5 \mathrm{~mm}, \mathrm{x}_{\mathrm{f}}=7.4 \mathrm{~mm}, \mathrm{y}_{\mathrm{f}}=10.1 \mathrm{~mm}\right)$

Fig. 2. shows how the array elements are matched to $50 \Omega$ (reflection coefficient: $\Gamma<-10 \mathrm{~dB}$ ) in a band wider than $100 \mathrm{MHz}$ around the $2.35 \mathrm{GHz}$ operating frequency and the isolation between two adjacent elements is larger than 20 dB.

The array is RHCP with an Axial Ratio (AR) below 3dB for all scanning angles and the main lobe has a measured gain of $9.2 \mathrm{dBi}$ with a $3 \mathrm{~dB}$ half beamwidth of $9^{\circ}$, which remains constant over the scanning range.

Scanning of the beam is handled by a beamforming circuit consisting of a microcontroller, a power divider and 4 phase shifters. The range of scanning angles is limited by the used phase shifters, manipulation of the main beam direction, without beam deformation, is achievable for all angles between $-15^{\circ}$ to $+15^{\circ}$.

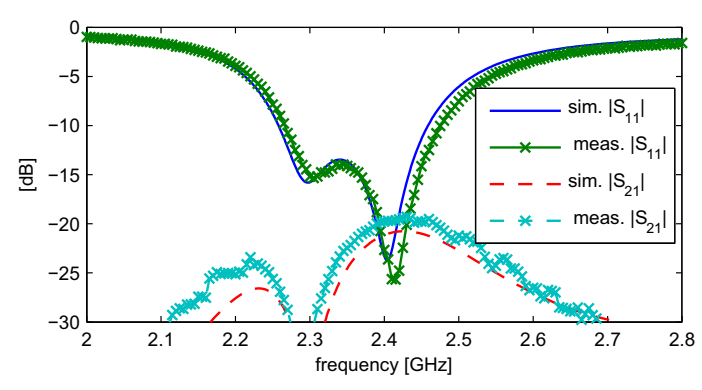

Fig. 2. Simulated (no marker) and measured (x-marker) reflection coefficient and mutual coupling between two neighboring array elements.

\section{B. Receiving Side}

The signal reflected back to the wearer is received by a left handed circularly polarized (LHCP) active receive antenna, which consists of a rectangular ring patch directly connected to a Low Noise Amplifier (LNA) underneath its ground plane.

By not using any matching networks between patch and LNA, losses related to the matching network are avoided and radar sensitivity is improved. The chosen antenna polarization increases the isolation between transmitter and receiver and minimizes the negative influence of secondorder reflections and multipath effects on the system performance.

The active antenna's topology and dimensions are depicted in Fig. 3. The LNA substrate is multilayered: the copper footprint is etched onto a $25 \mu \mathrm{m}$ polyimide film and glued on top of an Aramid layer. The active element is an ATF-54143 +e PHEMT from Avago technologies, applied in a grounded source topology [5]. This design provides a stable LNA with sufficient gain and linearity as well as a low noise figure. Like the passive transmit array, the antenna substrate is polyurethane foam and ground plane and patch are cut out of Flectron.

The active antenna has a measured gain of $15.7 \mathrm{dBi}$ and noise figure of $1.1 \mathrm{~dB}$. The measured $3 \mathrm{~dB}$ beamwidth exceeds $60^{\circ}$ and the $3 \mathrm{~dB}$ axial ratio beamwidth in terms of circular polarization is larger than $50^{\circ}$.

\section{Data processing}

Moving targets are detected by adopting a time multiplexing approach, where the transmit array scans the surroundings at a discrete number of directions. Scanning occurs at high speed, i.e. the period of a single sweep is small compared to the period of the received Doppler signal. Each sweep, the Doppler signal is sampled, with every 
sample corresponding to a discrete transmit angle. By combining the samples of the subsequent scans for each of the directions, waveforms are formed. Each waveform corresponds to the received signal from a single direction and it is as if all the different directions are scanned "simultaneously." The sweep frequency and the number of discrete sampling angles limit the maximal detectable Doppler shift. For detection of walking humans, this upper frequency does not cause problems, since the Doppler shift remains sufficiently low.

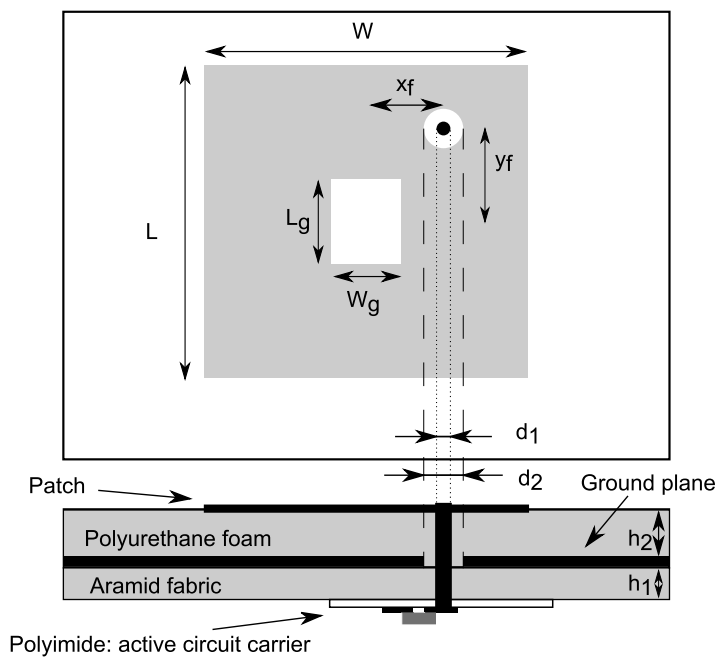

Fig. 3. Active antenna topology $\left(\mathrm{W}=49.5 \mathrm{~mm}, \mathrm{~L}=51 \mathrm{~mm}, \mathrm{~W}_{\mathrm{g}}=17.5 \mathrm{~mm}, \mathrm{~L}_{\mathrm{g}}=7 \mathrm{~mm}\right.$, $\left.\mathrm{x}_{\mathrm{f}}=8 \mathrm{~mm}, \mathrm{y}_{\mathrm{f}}=-5 \mathrm{~mm}, \mathrm{~h}_{1}=0.4 \mathrm{~mm}, \mathrm{~h}_{2}=3.55 \mathrm{~mm}\right)$

\section{MEASUREMENTS AND RESUlTS}

Measurements, with a person wearing the radar system, are performed to validate the system performance. The transmit array is attached onto the user's chest and the active receive antenna is placed on the stomach far enough from the transmitter to guarantee good isolation between both. Beamforming circuitry is attached to the back of the suit.

Two scenarios are considered, one in absence of a barrier and one 'through-wall scenario' with a barrier. Audio speakers, covered with copper tape and playing a low frequency tone, are used to create a repeatable moving object.

For the first measurement, a speaker playing a $20 \mathrm{~Hz}$ tone, is placed at an angle of $-15^{\circ}, 3 \mathrm{~m}$ from the radar and worn by a person. Fig. 4(a) shows how the object is detected at the $-15^{\circ}$ angle. In the second test, a speaker is placed behind a door covered by fire retardant paint. The speaker now plays a $15 \mathrm{~Hz}$ tone, distance between radar and object is $3 \mathrm{~m}$ and the angle $0^{\circ}$. The radar spectrogram for this measurement is shown in Fig. 4(b). The presence of the barrier does not limit the radar's ability to detect the moving object.

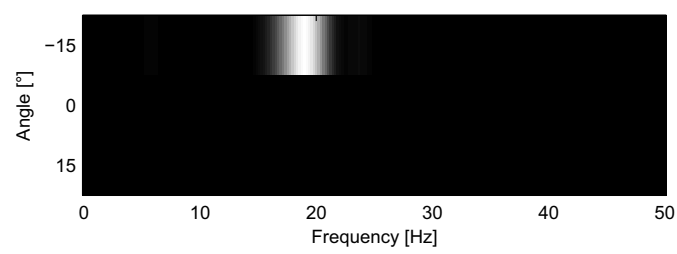

(a)

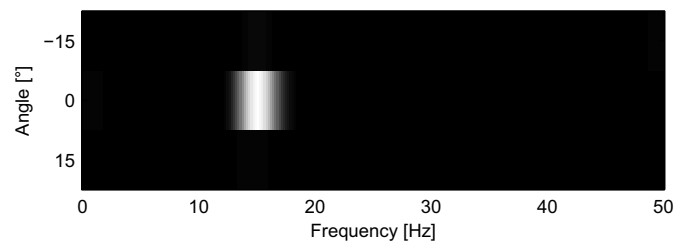

(b)

Fig. 4. On-body radar spectrogram for (a) a speaker placed at $3 \mathrm{~m}$ distance, producing a $20 \mathrm{~Hz}$ audio tone (b) a speaker at distance of $3 \mathrm{~m}$, producing a $15 \mathrm{~Hz}$ tone, behind a closed door.

\section{CONCLUSIONS}

In this contribution, a dedicated design approach is presented that relies on off-the-shelf textile materials, commonly found in protective garments to implement a wearable radar system, extending the functionality of rescue worker gear.

A textile RHCP transmit array is used to scan the surroundings and a LHCP active receive antenna, optimized for noise performance, is used to enhance the system sensitivity. The opposing polarizations enhance isolation between the on-body antennas and allows for easy rejection of second-order reflections.

Measurements have validated that the system, worn by a person, is capable of detecting moving objects in indoor environment, including a 'through-wall' scenario.

\section{REFERENCES}

[1] E. J. Baranoski, "Through-wall imaging: historical perspective and future directions," Journal of the Franklin Institute, vol. 345, no. 6, pp. 556-569, 2008.

[2] Agneessens, S., Van Torre, P., Declercq, F., Spinnewyn, B., Stockman, G. J., Rogier, H., Vande Ginste, D., "Design of a Wearable, Low-Cost, Through-Wall Doppler Radar System," International Journal of Antennas and Propagation, vol. 2012, Article ID 840924, 9 pages, 2012.

[3] Declercq, F., Rogier, H., and Hertleer, C.: 'Permittivity and loss tangent characterization for garment antennas based on a new matrix-pencil two-line method', IEEE Trans. Antennas Propag., 2008, 56, (8), pp. 2548-2554.

[4] M. L. Scarpello, D. Vande Ginste, and H. Rogier, "Design of a low-cost steerable textile antenna array operating in varying relative humidity conditions," Microwave and Optical Technology Letters, vol. 54, no. 1, pp. 40-44, 2012.

[5] ATF-54143, "High Intercept Low Noise Amplifier for the 1850-1910 MHz PCS Band using the Enhancement Mode PHEMT," Application Note 1222, Avago Technologies, 2006. 


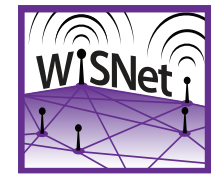

\section{PROCEEDINGS}

\section{Hub Page}

\section{IEEE Topical Conference on Wireless Sensors and Sensor Networks}

20-23 January 2013 - Austin, Texas, USA

\begin{tabular}{|l|l|}
\hline Message from the RWW 2013 General Chair & Welcome Page \\
\hline RWW 2013 Conference Chairs & Table of Contents \\
\hline RWW 2013 Technical Program & Author Index: Brief or Detailed \\
\hline Manuscripts \\
\hline
\end{tabular}

Proceedings of the 2013 IEEE Topical Conference on Wireless Sensors and Sensor Networks. For proceedings on USB: IEEE Catalog Number CFP13WST-USB, ISBN 978-1-4673-2931-6. For proceedings on CD: IEEE Catalog Number CFP13WST-CDR, ISBN 978-1-4673-2930-9. Copyright and Reprint Permission: Abstracting is permitted with credit to the source. Libraries are permitted to photocopy beyond the limit of U.S. copyright law for private use of patrons those articles in this volume that carry a code at the bottom of the first page, provided the per-copy fee indicated in the code is paid through Copyright Clearance Center, 222 Rosewood Drive, Danvers, MA 01923. For other copying, reprint or republication permission, write to IEEE Copyrights Manager, IEEE Operations Center, 445 Hoes Lane, Piscataway, NJ 08854. All rights reserved. Copyright (C2013 by IEEE. For technical support please contact Causal Productions (info@causalproductions.com).

Legend: 紧 View Manuscript 